\title{
An Integrated Laboratory for Collaborative Design in the Air Transportation System
}

\author{
Arne Bachmann ${ }^{1}$, Jesse Lakemeier ${ }^{1}$, Erwin Moerland ${ }^{1}$
}

\begin{abstract}
At the Institute of Air Transportation Systems of the German Aerospace Centre (DLR), methods for collaborative design are systematically developed and assessed. These collaborative design approaches are used to gain knowledge on the overall air transportation system. A collaborative working environment-the Integrated Design Laboratory (IDL) is established. It forms an experimental technical platform for integrating the competences of disciplinary experts within DLR. Within the laboratory, technical solutions, collaboration methodologies and organisation of teamwork are provided and evaluated to enhance multimodal communication between specialists. In this paper, experiences from previous DLR projects as well as observations on similar facilities are used to identify research areas. In a pilot study, requirements for the design room are laid out. The initial setup of the laboratory is presented, after which a research roadmap for enhancing collaborative design at DLR is presented.
\end{abstract}

\section{Keywords}

Aircraft Design, Air Transportation System, Collaborative Design, Concurrent Engineering, Multidisciplinary Design Optimisation

\section{Introduction}

The topics Concurrent Design (CD), Concurrent Engineering (CE) and Simultaneous Engineering (SE) have been subject of research since the 1990s $[1,20,24,33]$. These terms usually refer to a methodology that tries to integrate sequential design tasks into a more overlapping or even fully parallel organisation of tasks originating from different product development or lifecycle phases. In addition, a tighter coupling between disciplines results in higher interdisciplinarity, meaning an increased exchange of knowledge and application of trans-disciplinary methods. In concordance with [7], the main change when employing CE tech-

\footnotetext{
${ }^{1}$ A. Bachmann ( $\left.\square\right)$, J. Lakemeier, E. Moerland

Institute of Air Transportation Systems, DLR, Blohmstraße 18, 21079 Hamburg, Germany

e-mail: arne.bachmann@dlr.de
} 
niques is to reorganise existing tools, human resources and knowledge to increase overall efficiency of the design process. Upfront, parallel task organisation might lead to an increase in communicative effort and organisational overhead per discipline. This effort is, however, expected to reduce risks from errors that otherwise would be detected far later and thus lead to exponentially higher costs. Additionally, a reduction in development time and costs is usually expected after a period of seemingly slower but highly involved cooperation.

Although the original application for the "concurrent" approach stems from product design with manufacturing in mind, the idea of turning a sequential process into a parallel process has been adopted by many fields of research and development. These include the agile software development philosophy [8] and business processes [11]. In this paper we focus on the integrated evaluation and design of the air transportation system that is practised at the German Aerospace Centre (DLR), Germany's national research centre for aeronautics and space.

At DLR, the need for quick and valid assessment and studies in interdisciplinary project work is seen as a necessity to design the air traffic system of the future. Separation into individual disciplines is a commonly established method to decompose the complex design problems within aerospace sciences. With the current development of the Multidisciplinary Design Optimisation (MDO) approach, however, it is seen as a cultural shift to bring those increasingly detaching disciplines and technologies together, using improved knowledge management approaches for better communication between the disciplines [9]. These disciplines include, but are not limited to, aerodynamics, structural mechanics, engine design, and air traffic management. One challenge for any interdisciplinary approach comes from the fact that the sub-disciplines are concerned with different subject matters. For instance, aerodynamics concerns the flow of air around solid objects, while structural mechanics analyses deformations and forces in structures.

A second challenge comes from the multiple levels at which sub-disciplines may connect to each other. On one hand, the aforementioned two disciplines operate on the system level with regard to the aircraft. On the other hand, the aircraft is a subsystem with respect to air traffic management and environmental impact considerations. A holistic approach, therefore, needs to account for system-of-systems levels. While allowing to gain deep understanding of singular problems, the decomposition of the problem area "aerospace" into distinct disciplines is a major obstacle to integral knowledge of complex problems today [9].

In order to approach the complex analysis of the entire air transportation system holistically, the Institute of Air Transportation Systems at DLR focuses on the aggregation and integration of existing expert knowledge within DLR. Current investigations include design configurations for blended wing body and quiet short take-off and landing aircraft, but also development of noise abatement techniques and forecast of climate impact [22,23]. The ability to consider sub-system, systemof-systems, or global design levels is of paramount importance for performing overall assessments in aircraft design. 
For many projects at DLR the concurrency aspect of CE applies very well, since often many disciplines and stakeholders collaborate towards a common design goal simultaneously, while communication and coordination requirements are necessarily high throughout the projects. Our approach differs, however, from the idea of manufacturing since the results of design exercises are often virtual - there is usually no physical airplane to be built - and design projects are characterised by relatively long development times. The generated knowledge is used to assess scenarios, consult policymakers and to share knowledge with industrial partners and the scientific community. We suggest calling the highly integrated interdisciplinary design process employed in aerospace projects collaborative design.

To meet the challenging requirements which are posed by society towards the air transport system, trans-disciplinary integration is mandatory today [16]. Following the current trend, organisations tend to incorporate more and more disciplinary knowledge bearers into their design processes developing from intra- to interdisciplinary collaboration. The MDO methodologies progress from multidisciplinary to highly integrated interdisciplinary collaboration.

The best-known facilities and working groups that subsume under the CE label and concern applications in aerospace engineering are listed in Table 1. Our project iTALENT at DLR is engaged in constructing an experimental collaborative design environment called Integrated Design Lab (IDL). It is a place where experiments concerning collaboration are conducted. Rather than emphasising the service character of a facility, IDL serves systematic development and examination of improved methods for (concurrent and co-located) collaboration in the design of the air transport system. Additionally, it provides the technical infrastructure for enhanced communication between engineers and serves as a test bench for experimentation with collaboration methods. The following section describes the pilot study carried out in the IDL, which has led to the prototype platform described in the third section. The paper is concluded by a discussion and outlook.

Table 1: Interdisciplinary co-located design institutions in aerospace applications

\begin{tabular}{|c|c|c|c|c|c|c|}
\hline Name & Full name & Institution & Location & Founded & Focus & Ref. \\
\hline ASDL & $\begin{array}{l}\text { Aerospace Systems } \\
\text { Design Laboratory }\end{array}$ & $\begin{array}{l}\text { Georgia Tech School of } \\
\text { Aerospace Engineering }\end{array}$ & Atlanta (USA) & 1992 & aerospace systems and complex design & 2,27 \\
\hline PDC & Project Design Center & $\begin{array}{l}\text { NASA Jet Propulsion Laboratory } \\
\text { (JPL) }\end{array}$ & Los Angeles (USA) & 1994 & $\begin{array}{l}\text { develop, promote and support the } \\
\text { use of concurrent engineering } \\
\text { techniques by JPL design teams }\end{array}$ & 21,31 \\
\hline CDC & Concept Design Center & The Aerospace Corporation & El Segundo (USA) & 1997 & $\begin{array}{l}\text { rapid development of conceptual } \\
\text { designs for new space systems with } \\
\text { corporate engineering experts }\end{array}$ & 3,32 \\
\hline CDF & $\begin{array}{l}\text { Concurrent Design } \\
\text { Facility }\end{array}$ & ESA ESTEC & Noordwijk (NL) & 1998 & $\begin{array}{l}\text { facility allowing teams of experts to apply } \\
\text { concurrent engineering methods to the } \\
\text { design of future space missions }\end{array}$ & $\begin{array}{r}6,7 \\
12\end{array}$ \\
\hline IDC & $\begin{array}{l}\text { Integrated Design } \\
\text { Center }\end{array}$ & $\begin{array}{l}\text { NASA Goddard Space Flight } \\
\text { Center (GSFC) }\end{array}$ & Greenbelt (USA) & 2003 & $\begin{array}{l}\text { collaborative engineering facility for } \\
\text { space missions in all phases of design, } \\
\text { including a VR facility }\end{array}$ & 17,19 \\
\hline $\mathrm{CDL}$ & $\begin{array}{l}\text { Concurrent Design } \\
\text { Laboratory }\end{array}$ & York University & Toronto (CA) & 2006 & $\begin{array}{l}\text { support for the Space Mission Analysis } \\
\text { and Design course at York University }\end{array}$ & 13,26 \\
\hline CEF & $\begin{array}{l}\text { Concurrent Engineering } \\
\text { Facility }\end{array}$ & $\begin{array}{l}\text { DLR System Analysis Space } \\
\text { Segment (SARA) }\end{array}$ & Bremen (GER) & 2008 & $\begin{array}{l}\text { feasibility studies in the conceptual } \\
\text { phase for space systems and missions, } \\
\text { transfer to other fields foreseen }\end{array}$ & $\begin{array}{r}14, \\
29,30\end{array}$ \\
\hline IESTA & $\begin{array}{l}\text { Infrastructure for Evaluat- } \\
\text { ing Air Transport Systems }\end{array}$ & $\begin{array}{l}\text { ONERA Long-term Design \& } \\
\text { Systems Integration Department }\end{array}$ & Toulouse (FR) & 2008 & $\begin{array}{l}\text { assessing the impact of new } \\
\text { technologies air transportation system }\end{array}$ & 15,18 \\
\hline
\end{tabular}




\section{Pilot Study}

After introducing the current state of multidisciplinary collaboration within DLR, the current section presents the overall goals of the pilot study being performed in the IDL and shows the general requirements on the laboratory.

\subsection{Background}

To get to a point of highly integrated multidisciplinary collaborative design, the following phases can be identified:

- The first phase consists of the core development of specialised analysis tools. This development is often driven by disciplinary needs and interests. Collaboration is possible by offering access to the software program through the source code, an executable file or a remote network access. Administration of individual tools is decentralised. The responsible departments thereby maintain sovereignty over their code.

- The second phase crystallised during former DLR projects and concerns the creation of common interfaces between disciplinary tools. Since interface is a very generic term, the approach taken in the projects was to define a common data format which is used to store and transfer data via lower-level data interfaces $[4,5,25]$. The XML-based Common Parametric Aircraft Configuration Schema (CPACS) is nowadays used to foster data exchange between disciplinary analysis tools.

- The third phase is the meaningful combination and interpretation of aggregated workflows. After combining the disciplinary software tools in workflows, both the overall and individual results generated by the workflow components are validated. A plausible discussion and verification of generated results requires a certain process and maturity of a working group's understanding of the overall field of investigation. The term Multidisciplinary Design Analysis and Optimisation (MDAO), coined by NASA, emphasises the actual analysis of the overall system, the understanding of the problem, and the process to its creation.

- The fourth phase in integrated design is formed by the application of a collaborative design workflow to find solutions for a specific design exercise; this level can be seen as a concurrent design session. During such a session, disciplinary experts gather in the IDL to monitor disciplinary results and bring in their disciplinary expertise. A major investigation concerns finding efficient methods of communication between these experts.

In future (fifth phase) projects, knowledge gained from levels three and four can be reintroduced into core development activities to improve and accelerate development. While in earlier interdisciplinary collaboration projects at DLR focus was mainly put on the first and second phase of design collaboration, the current pro- 
ject Virtual Aircraft Multidisciplinary Analysis and Design Process (VAMP) aims at creating an improved understanding of the collaborative build-up process, advancing towards the ability to collaboratively conduct novel aircraft design exercises. During the VAMP project, creating a platform for collaborative research is targeted. Aside from extending technical interfacing possibilities, focus is also on gaining experience on implementing collaboration processes on an expert level. To aid in these advancements, the iTALENT project functions as the experimental hub to leverage experimentation of collaborative factors like visualisation, group seating arrangement, moderation of work sessions and communication in general.

\subsection{Requirements}

The origin of the present study is the elicitation and definition of requirements for a multidisciplinary collaborative laboratory that lead to the construction of the IDL. Several usage scenarios have been defined and analysed regarding a previously defined set of feature categories. The selection and type of features considered is certainly arbitrary and not necessarily exhaustive, but nevertheless useful because they lead to verifiable requirements:

- type of video signal (output, distribution, input)

- layout of visualisation / type of contents to stream

- available software collection

- remote software and remote desktop access

- logging and recording

- data exchange and storage

- spatial range of motion and interaction
- usage of displays - connection type and signal no.

- network connectivity, security and privacy

- support for spontaneous software installations

- mobile computing and input support

- conduct and moderation

- seating arrangement

- necessary roles of supporting personnel

The list puts primarily a focus on technical aspects concerning the room design, lab infrastructure and equipment. For each feature, a rough solution space was created and options for implementation were investigated. A morphological analysis was performed by reflecting the requirements during identified usage scenarios, in order to obtain the most suitable combination of laboratory feature options.

Non-technical requirements concern, e.g., the organisation of collaborative work, and often cut across several of the technical aspects and cannot be separated or validated that easily. Expected gains and features of these also cannot easily be predicted but will evolve and develop over time when practical knowledge in experimental design sessions is gathered. Among these somewhat vaguely defined requirements are:

- improving interdisciplinary communication

- accelerating decision making processes during conceptual studies

- improving integration methods with external partners
- improving (practical) knowledge management

- developing visualisation modes for interdisciplinary knowledge exchange

- offering integration services and supporting partners in efficient model integration 
In order to arrive at a laboratory in which systematic experimentation is possible, the (technical) requirements for its design are described. Thereafter some preliminary results of initial collaborative design sessions are presented.

Visualisation is a powerful tool for collaboration and knowledge transfer. In order to work collaboratively on projects concerning the entire air transportation system, numerous different visualisation contents have to be displayed simultaneously. For instance, 3D-drawings of concept aircraft, data tables displaying physical properties of the design, world maps showing route networks and aircraft movements, plots of costs or emissions and so on.

Beside a large screen size and omnipresent presentation possibilities, an important factor is to use the available space efficiently for the given use case. The visualization capabilities are important for the participants to integrate their results, which are computed beforehand or developed during the design session on their own computers. Therefore, a hardware and software environment will be established., This allows not only to display global contents from the host computer, but also data coming in from connected mobile, remote or stationary computers of all participants.

Network. Solutions regarding network infrastructure are widely available. However, when collaborating with external partners, the problem of security, trust and adjustable access to mutual data emerges. A central visualisation server, which has several network adapters, provides a single point of administration. This makes network separation and security administration manageable.

Seating arrangement needs to adapt to the specific mode of interaction, which varies according to the specific problem. Furthermore, collaboration methods will evolve over time, so a flexible configurable approach to seat and desk positions is best suited for the IDL. For example, a half-circular arrangement in front of a large display area as used in general control rooms could be advantageous for communication and viewing perspective. This, however, only works for a limited number of participants since face to face distances might become too large. Alternatively, if the communication between the attendees is of higher priority than unobstructed sight, a horseshoe configuration can be more suitable due to the small communication distances between attendees. Also a configuration with multiple rows of seats should be possible in case of presentations in front of a big audience. Finally, for more focused work groups, an "island" configuration could be the best choice. This arrangement is applied, e.g., in the ASDL (Table 1) and facilitates communication within small groups, but might discourage communication with users of other table groups.

Requirements derived from the seating considerations include the creation of work spaces which can easily and quickly be rearranged without re-plugging lots of cables. The system must also be modular, as the number of required work spaces varies from application to application.

Knowledge management. Properly managing the generated knowledge is of paramount importance during the design of complex systems. Especially due to 
the current trend in ever increasing available computational power, large amounts of data are generated and need to be properly interpreted and processed. Knowledge of an explicit nature can be saved and - where possible - converted into design rules. By programming these design rules in a flexible design system, repetitive tasks can be automated and more time is left to the engineers for creative design tasks [28]. Especially during these creative tasks, an important factor is managing tacit or implicit knowledge of the disciplinary experts involved. As part of ongoing investigations, the application of practical knowledge management methodologies and guidelines for moderators aid in executing the identified fourth phase of multidisciplinary collaborative design. Since it is foreseen to execute unconventional air transportation system design tasks within the IDL, efficiently integrating expert observations and opinions in the design process is an important requirement. During practical application sessions within the IDL, the required knowledge to be provided and its corresponding visual form will be identified. Finding a proper balance between flexibility and performance of the knowledge provision on the one hand, and providing the right transparency and data security on the other hand is therein required.

According to the exemplary technical and non-technical requirements, a prototypical infrastructure for the IDL has been derived. As shown in Figure 1, participants are able to connect with each other and with the central system through various input connections, both local and remote. Access to central computing resources is provided, and a moderator may have control over the workflow steering and the data to display. The connection to a common visualisation wall is shown at the bottom part of the figure.

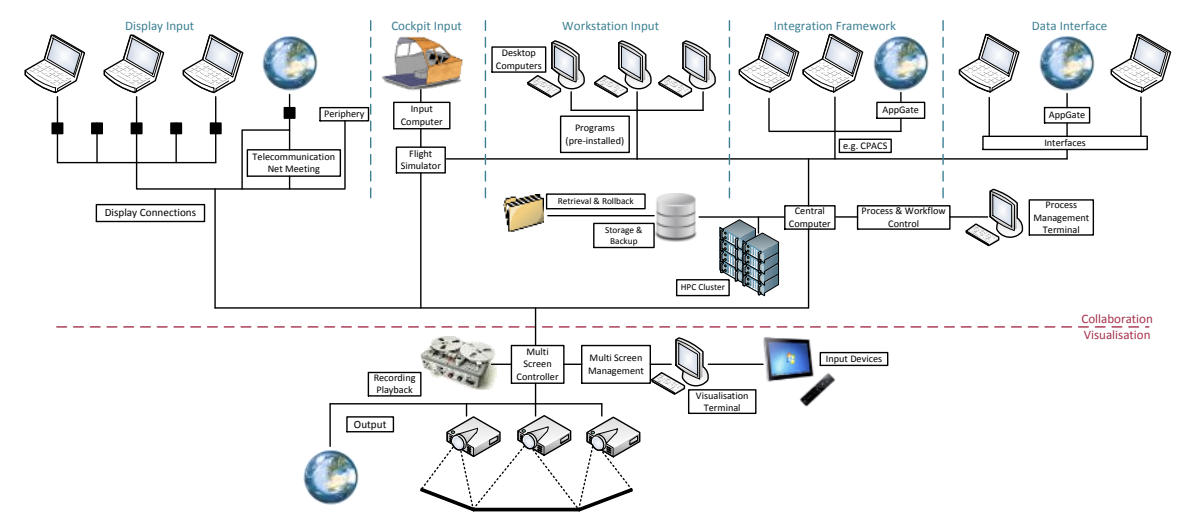

Figure 1 Schematic of the IDL's technical infrastructure 


\section{Prototype}

The first stage of the Integrated Design Laboratory, the so-called basic platform, has been built up inside an office building in Hamburg-Harburg.

Beside the main design room, the laboratory is equipped with an additional meeting room for discussions within smaller groups, and a lobby to welcome and cater guests, as well as for the important socialising part of gatherings. This "lounge" strives to provide a more relaxed atmosphere, in contrast to the workoriented efficient atmosphere emanating from the design room. Figure $\mathbf{2}$ shows the IDL room layout schematically. The implementation of the basic platform has been realised according to the requirements identified beforehand.

Visualisation. The IDL prototype uses a mobile preliminary visualisation wall to allow for the evaluation of different geometrical constellations. The front projection system is divided into three separate screens with a total size of $10 \times 2.25 \mathrm{~m}$.

To share screen content with all attendees on the visualisation wall, different hardware and software solutions are considered. A mainly hardware-oriented solution is found in transmitting the VGA or HDMI signals from participants' laptops to a central visualisation computer. This solution does not require any hardware or software modifications on the participants' laptops, but requires dedicated hardware and software on the visualization computer. Since either no contents or the complete screen can be shared, this is not considered to be the most flexible solution. Another approach is not to hardwire the display output of participants, but to stream (partial) screen contents via network connections. This solution requires dedicated software to be installed on participants' laptops as well as on the visualisation computer. In return it does not require any dedicated hardware, provided that all computers have a sufficiently fast network connection and allows for sharing partial screen contents.

In case of the IDL, a combination of both approaches is applied. In general, the second approach is implemented, using a commercial software suite of a major display hardware manufacturer. With the help of A/D converters able of transferring VGA or HDMI signals via Ethernet, computers which for security reasons cannot be connected to the network may still share their visual data.

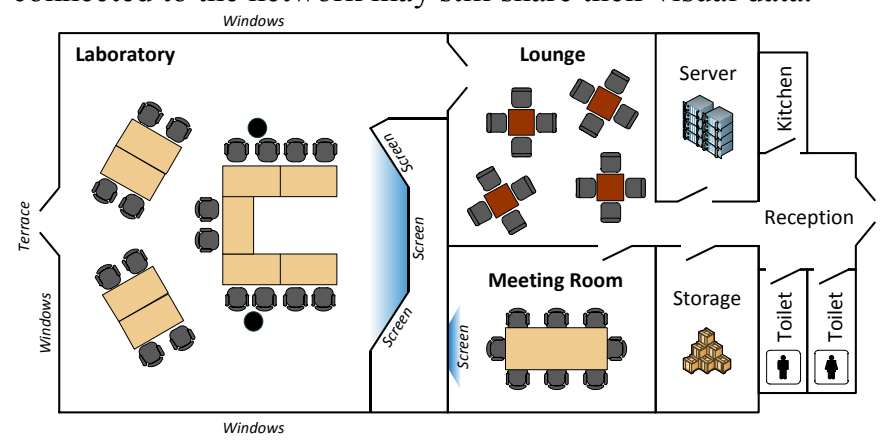

Figure 2: Floor plan of the Integrated Design Laboratory 

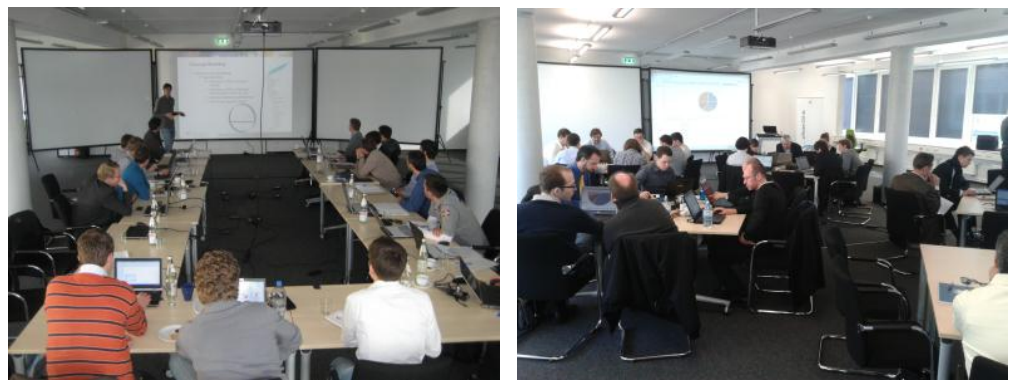

Figure 3: Design Session inside the IDL basic platform with (A) horseshoe and (B) islands seating arrangement.

Seating arrangement. For the workplaces of the prototype laboratory, movable desks for one or two participants have been chosen. This is considered to be the most flexible and economical way to start performing exploratory design sessions. Desk weight and seating comfort were considered, and desks were equipped with rollers. There is one tiltable and turnable monitor per desk which can be used by each of the desk users in addition to their individual laptops.

A more demanding aspect in this context is realising a cable management, suitable for performing ad-hoc changes in the arrangement, without the need to replug dozens of power, Ethernet and image transmission cables. The concept realised inside the IDL is to build up a daisy chain between the tables, starting from central hubs located at the two pillars in the middle and at the front side of the room. Each table has inputs for power and Ethernet on one side and outlets build right into the table surface. Power strips and Gigabit switches are installed under each table and provide ports to the attendees via table tanks and also provided as outputs on the other side of the table.

This concept allows placing the tables anywhere in the room, connected by a single wiring harness. It significantly reduces cable clutter throughout the room as well as the time effort for changing the arrangement. However, considerations of transmission performance have to be taken into account, when continuously streaming large amounts of data.

Further conceptual work regarding network and knowledge management requirements mentioned in the previous section will be approached within the upcoming project phases.

Preliminary test cases and future studies. During the intermediate VAMP project meeting held in the IDL in April 2012, general consensus on the application of the latest major upgrade of the CPACS data exchange format was required (cf. Section 2). Therefore, a group-oriented horseshoe seating arrangement was chosen (cf. Figure 3A), promoting efficient plenary discussions. The visualisation wall was used for guiding plenary discussions, among others by using illustrative presentations and flexible mind-mapping software. The interactive involvement of the participants has contributed to the efficient and effective establishment of consensus, and was positively experienced. A design session focusing on the applica- 
tion of a distributed collaborative workflow environment for solving engineering problems was held in an "islands" seating arrangement, to promote bilateral interactions (cf. Figure 3B). The visualisation wall was used for showing presentations, software applications and overall calculation results. The threshold for data exchange and communication appears to be relatively low using this setup. During upcoming meetings, more seating arrangements and visualisation methodologies will be examined and compared to gain experience on efficient collaboration.

The next meeting of the VAMP project will be held at the end of 2012, in which - aside recording the lessons learned during the collaboration project - the generated collaborative design workflow will be applied to obtain an initial design for a quiet short range aircraft. Disciplines explicitly included in the design are aerodynamics, structures, landing gear design, stability analysis, mission simulation, noise and pollution analysis, and engine analysis. Remaining disciplines in aircraft design are currently covered by the in-house developed conceptual aircraft design tool VAMPzero [10]. During the design session, an important focus will be placed on creating mutual understanding of involved disciplines, by effectively using visualisation possibilities and interaction methods within the IDL.

Another upcoming project meeting inside the IDL will be the review meeting for the project Climate-compatible Air Transport System (CATS) [22,23], scheduled for October 2012. Within this project, the climate impact of a certain aircraft has been analysed for a large amount of flight routes. Different operating points of Mach numbers and cruise altitudes have been simulated to determine the optimal trade-off between climate impact and operation costs.

The review meeting is of special interest for the IDL development, as it will be the first meeting where a specifically programmed visualisation application developed for the lab will be applied. This visualization application contains graphical user interfaces to navigate through routes on a world map and plot detailed simulation results for certain routes and trajectories. It also contains an option to visualise single or multiple simulated missions. The multi-level concept of the IDL visualisation application is outlined in Figure 4. It is planned to extend the visualisation application and to apply it for other project or review meetings.

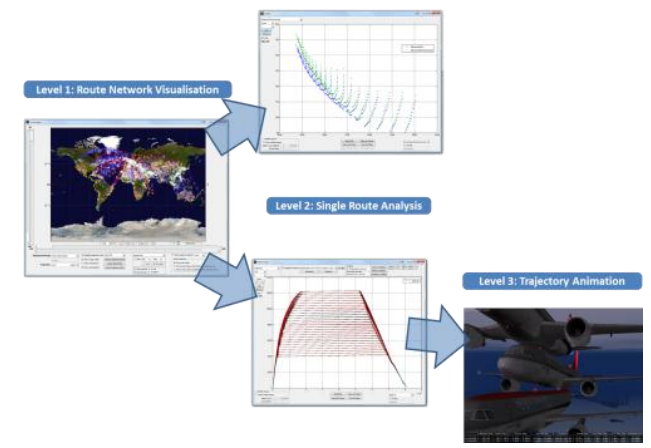

Figure 4: Multi-level approach of the IDL visualisation application 


\section{Conclusion and Outlook}

The basic platform concept of the Integrated Design Laboratory has been presented. In the IDL, an increase in collaboration efficiency is achieved by leveraging visual media, reducing communication barriers and applying collaborative design methods in a teamwork-oriented environment.

By the end of 2012, the basic platform of the IDL will be upgraded into a second stage, the more advanced research platform. With this stage, the preliminary visualisation wall will be replaced by a high-end solution, removing the wellknown disadvantages of front projections and thus allowing project work with external partners as well. The third stage of IDL, the application platform, will be introduced in 2013 and targets an overall finalised state concerning mechanical and electrical installations, as well as provided software and hardware. In addition to the progress regarding technical requirements, the focus of further studies will be mainly on the improvement of collaboration and design methodologies that build upon this progress.

\section{Acknowledgements}

The iTALENT project is funded by the Agency of Economy, Transport and Innovation (BWVI) of the Free and Hanseatic City of Hamburg, through grant LAHH143 of the second aviation research programme of Hamburg. We are grateful to Dr. Evelina Dineva for her collaboration during preliminary investigations.

\section{References}

1. Adler, R. E. \& Ishii, K. (1989) DAISIE: Designer's aId for sImultaneous engineering. In: Proceedings of the 1989 ASME International Computers in Engineering Conference and Exposition (pp. 19-26). Anheim, CA, USA: American Society of Mechanical Engineers (ASME), New York, NY, USA.

2. Aerospace Systems Design Laboratory (ASDL) website (2012) http://www.asdl.gatech.edu

3. Aguilar, J. A., Dawdy, A. B., \& Law, G. W. (1998) The aerospace corporation's concept design center. In: 8th Annual International Symposium of the International Council on Systems Engineering, volume 2

4. Bachmann, A., Kunde, M., Litz, M., Böhnke, D., \& König, S. (2010) Advances and work in progress in aerospace predesign data exchange, validation and software integration at the German Aerospace Centre. In: Product Data Exchange Workshop 2010 Oslo, Norway

5. Bachmann, A., Kunde, M., Litz, M., \& Schreiber, A. (2010) Advances in generalization and decoupling of software parts in a scientific simulation workflow system. In: ADVCOMP 2010 Florence, Italia

6. Bandecchi, M. (2002) The ESA Concurrent Design Facility (CDF). http://s.dlr.de/w759

7. Bandecchi, M. (2007) Concurrent engineering at ESA: From the concurrent design facility (CDF) to a distributed virtual facility. In: The 14th ISPE International Conference on Concurrent Engineering (CE2007) Sao Jose' dos Campos, SP, Brazil 
8. Beck, K. \& et al. (2001) Manifesto for agile software development. http://agilemanifesto.org

9. Belie, R. (2002) Non-technical barriers to multidisciplinary optimization in the aerospace industry. In: 9th AIAA/ISSMO Symposium on MDAO (pp. 1-6). Atlanta, GA, USA

10. Böhnke, D., Nagel, B., \& Gollnick, V. (2011) An approach to multi-fidelity in conceptual aircraft design in distributed design environments. In Aerospace Conference (pp. 1-10), IEEE

11. Chitale, A. K. \& Gupta, R. C. (2007) Product Design and Manufacturing, 4th ed. Prentice Hall of India

12. Concurrent Design Facility (CDF) website (2006) http://www.esa.int/esaMI/CDF/

13. Concurrent Design Laboratory (CDL) website (2012) http://s.dlr.de/4c6s

14. Concurrent Engineering Facility (CEF) website (2012) http://s.dlr.de/w9w2

15. Elie, A., Kervarc, R., Dubot, T., \& Bourrely, J. (2008) IESTA: A modular distributed simulation platform for the evaluation of air transport systems. In: MASCOT08-IMACS/ISGG Workshop

16. Flightpath 2050. Europe's vision for aviation (2011)

17. Gough, K. M., Allen, B. D., \& Amundsen, R. M. (2005) Collaborative mission design at NASA Langley research center. http://hdl.handle.net/1853/8020

18. Infrastructure for Evaluating Air Transport Systems (IESTA) website (2012) http://www.onera.fr/iesta/

19. Integrated Design Center (IDC) website (2011) http://idc.larc.nasa.gov/

20. Ishii, K., Adler, R. E., \& Barkan, P. (1988) Application of design compatibility analysis to simultaneous engineering. In: Artificial Intelligence for Engineering, Design, Analysis and Manufacturing, volume 2 (pp. 53-65). http://s.dlr.de/515z

21. Jet Propulsion Laboratory (JPL): Team X website (2007) http://jplteamx.jpl.nasa.gov

22. Koch, A., Dahlmann, K., Grewe, V., Kärcher, B., Schumann, U., Gollnick, V., \& Nagel, B. (2009) Integrated analysis and design environment for a climate compatible air transport system. In: 9th AIAA Aviation Technology, Integration, and Operations Conference (ATIO) Hilton Head, SC, USA

23. Koch, A., Lührs, B., Dahlmann, K., Linke, F., Grewe, V., Litz, M., Plohr, M., Schumann, U., Gollnick, V., \& Nagel, B. (2011) Climate impact assessment of varying cruise flight altitudes applying the CATS simulation approach. In: 3rd International Conference of the European Aerospace Societies (CEAS) Venice, Italy

24. Kusiak, A. \& Park, K. (1990) Concurrent design: Decomposition of design activities. In: 2nd International Conference on Computer Integrated Manufacturing (pp. 557-563). Troy, New York: IEEE

25. Liersch, C. M. \& Hepperle, M. (2009) A unified approach for multidisciplinary preliminary aircraft design. In: CEAS 2009 European Air and Space Conference Manchester, UK

26. Minisci, E. \& Vasile, M. (2006) Concurrent design lab in Glasgow. http://s.dlr.de/nybt

27. Osburg, J. \& Mavris, D. (2005) A collaborative design environment to support multidisciplinary conceptual systems design. SAE transactions, 114, pp. 1508-1516

28. Rocca, G. L. \& van Tooren, M. J. L. (2010) Knowledge-based engineering to support aircraft multidisciplinary design and optimization. In: Proceedings of the Institution of Mechanical Engineers, Part G: Journal of Aerospace Engineering, 224(9), p. 1041

29. Romberg, O., Braukhane, A., \& Schumann, H. (2008) Status of the concurrent engineering facility at DLR Bremen. In: DGLR-Congress Darmstadt, Germany

30. Schubert, D., Romberg, O., Kurowski, S., Gurtuna, O., Prévor, A., \& Savedra-Criado, G. (2010) A new knowledge management system for concurrent engineering facilities

31. Smith, J. (1998) Concurrent engineering in the Jet Propulsion Laboratory project design center. In: SAE CONFERENCE PROCEEDINGS P (pp. 165-178).: Society of Automotive Engineers, Inc.

32. Smith, P. L., Dawdy, A. B., Trafton, T. W., Novak, R. G., \& Presley, S. P. (2001) Concurrent design at aerospace. http://s.dlr.de/7743

33. Winner, R. I. (1988) The role of concurrent engineering in weapons system acquisition. Technical Report IDA Report R-338, Institute for Defence Analysis, Alexandria, VA, USA 